\title{
Sources of interference in the attentional blink: Target-distractor similarity revisited
}

\author{
WILLIAM S. MAKI, GIULIA BUSSARD, KELLIE LOPEZ, and BETH DIGBY \\ Texas Tech University, Lubbock, Texas
}

\begin{abstract}
Observers monitored streams of words or letters $(10 \mathrm{items} / \mathrm{sec})$ for one or two targets. An attentional blink (AB) effect was observed in which identification of the first target temporarily impaired identification of the second target. Target identification was impaired when the distractors were composed of either letters or false-font characters (cf. Maki, Couture, Frigen, \& Lien, 1997). An asymmetrical AB effect was observed with letters and mathematical symbols; the AB effect was largest for symbol targets and letter distractors. The characters used in these experiments were rated on their meaningfulness, familiarity, and other stimulus properties. The rating data showed that pixel density best accounted for the asymmetrical target-distractor similarity effects. Modulation of the $\mathrm{AB}$ effect by target-distractor similarity appears to result partly from low-level masking. But masking effects may be reduced by attentional capture by target features.
\end{abstract}

In the present research, rapid serial visual presentation (RSVP) was used to investigate a phenomenon known as the attentional blink (AB). The AB appears to have been discovered in different contexts by Broadbent and Broadbent (1987) and Sperling (e.g., Weichselgartner \& Sperling, 1987). Later, the AB was so named by Raymond, Shapiro, and Arnell (1992). The AB is demonstrated when an observer searches for two targets in an RSVP stream of to-be-ignored distractors. Detection and identification of the second target (T2) is impaired during a window spanning about 200-500 msec following presentation of the first target (T1). Often the AB is marked by a U-shaped function relating $\mathrm{T} 2$ performance to the lag between targets. ${ }^{1}$

The AB effect is robust. It has been shown when targets are words (Broadbent \& Broadbent, 1987; Maki, Couture, Frigen, \& Lien, 1997) and when targets are single letters (Chun \& Potter, 1995; Raymond et al., 1992). The U-shaped AB function survives manipulations at the time of test and appears for both recognition and recall tests (Maki, Couture, et al., 1997). The impairment in T2 performance occurs when targets and distractors occur in a single location (Raymond et al., 1992) and also when targets occur in multiple locations (Duncan, Ward, \& Shapiro, 1994; Weichselgartner \& Sperling, 1987). These studies included control conditions in which observers were instructed to ignore $\mathrm{T} 1$ (or T1 was omitted from the RSVP stream); performance was improved in the control condition, and the U-shaped function characterizing the

Experiments 1-4 were summarized at the 41st Annual Meeting of the Psychonomic Society, New Orleans, November 17, 2000. We thank Vincent Di Lollo and Mary Potter for helpful comments on earlier versions of this report. Correspondence concerning this article should be directed to W. S. Maki, Department of Psychology, Texas Tech University, Lubbock, TX 79409-2051 (e-mail: bill.maki@ttu.edu).
$\mathrm{AB}$ effect was flattened and displaced upward. The difference between the consequences of attending to both $\mathrm{T} 1$ and $\mathrm{T} 2$ and attending to $\mathrm{T} 2$ only shows that the $\mathrm{AB}$ effect is not merely the result of the perceptual difficulties of reporting a target from an RSVP stream; attending to the first target seems to be a necessary condition for producing the $\mathrm{AB}$ effect.

Chun and Potter (1995) proposed a two-stage model of the AB. In Stage 1, all items are claimed to enter a labile memory analogous to the conceptual short-term memory proposed by Potter (1993). If a target feature is detected, an item may then be processed further in Stage 2. The second stage in Chun and Potter's model is a limited-capacity process that converts items in Stage 1 into representations available to conscious awareness and verbal report (short term consolidation; Jolicœur, 1998). While Stage 2 is occupied with one item, subsequent items that enter the Stage 1 memory are susceptible to loss through interference from subsequent items.

Chun and Potter's (1995) model has been supported by subsequent studies employing both behavioral and eventrelated potential (ERP) techniques. The magnitude of the $\mathrm{AB}$ is known to depend on T1's being masked by a following distractor (Grandison, Ghirardelli, \& Egeth, 1997; Raymond et al., 1992; Seiffert \& Di Lollo, 1997). The AB is also determined by T2's being masked, or corrupted, by the next distractor (Giesbrecht \& Di Lollo, 1998; Maki, Couture, et al., 1997). The P3 component of the ERP, presumed to reflect the postperceptual updating of working memory, is suppressed during the $\mathrm{AB}$ (Vogel \& Luck, 2002). Semantic priming and ERP studies converge on the conclusion that word meaning is available during the AB (Maki, Frigen, \& Paulson, 1997; Shapiro, Caldwell, \& Sorensen, 1997; Shapiro, Driver, Ward, \& Sorensen 1997; Vogel, Luck, \& Shapiro, 1998). The implication of these studies is that the locus of the $A B$ is 
some postperceptual, limited-capacity process like Chun and Potter's Stage 2. However, knowing that some central processor is occupied with $\mathrm{T} 1$ and misses $\mathrm{T} 2$ does not explain exactly how T2 is lost (at least, lost to conscious report) when it falls within the $200-500 \mathrm{msec}$ window following $\mathrm{T} 1$.

Three different views of the fate of $\mathrm{T} 2$ have been proposed: overwriting, whole object substitution, and partial replacement. "Because representations in Stage 1 are ephemeral and rapidly overwritten by subsequent items, response features for T2 appearing during bottleneck processing of T1 will be overwritten by post-T2 items" (Chun, 1997, p. 1197; see also Jiang \& Chun, 2001). Although generally agreeing with Chun's two-stage interpretation of the $\mathrm{AB}$ effect, for other theoretical reasons (Di Lollo, Enns, \& Rensick, 2000), Brehaut, Enns, and Di Lollo (1999, p. 1446) "prefer the concept of [object] substitution to that of interruption or even overwriting" and explain that "substitution is intended to convey the sense that the original item has been completely replaced by the temporally trailing item, at least as far as conscious processes are concerned." Furthermore, Giesbrecht and Di Lollo (1998) reported that a dot mask following T2 was sufficient to produce an $A B$ and interpreted that finding as an instance of object substitution. However, there are data that are not compatible with these interpretations of the AB. Maki, Couture, et al. (1997) showed that the verbal report of $\mathrm{T} 2$ was often corrupted by the distractor immediately following T2 $(\mathrm{T} 2+1)$. For example, the T2 word BLOOD followed by the nonword distractor JANK was reported as BLANK. This fits with the two-stage model's focus on the events following T2 as sources of interference but does not square with the idea that $\mathrm{T} 2+1$ completely replaces $\mathrm{T} 2$ in Stage 1 memory and is thus misreported as T2. Instead, these blendings of $\mathrm{T} 2$ and $\mathrm{T} 2+1$ indicate that parts of $\mathrm{T} 2$ may be corrupted by (or replaced by parts of) subsequent distractors.

These differing accounts of the fate of $\mathrm{T} 2$ point to the need for a better understanding of the contribution of distractors to the $\mathrm{AB}$. Questions about the role of distractors have motivated research in which target-distractor (T-D) similarity has been investigated. The letters shown in Figure 1A typically have been used as targets, and the other character sets shown in Figure 1A have been used as distractors. Chun and Potter (1995) displayed two of the letters as targets in a stream consisting of either digits or mathematical symbols. They found a larger AB effect when letters were searched for in digits than when letters were searched for in symbols. Presumably, the letter targets were visually or categorically less similar to symbols than to digits. Maki, Couture, et al. (1997) created lists of words, pronounceable nonwords, consonant strings, and strings composed of false-font characters, all printed in black (Figure 1B); the targets were two words printed in red and green. The false-font characters were composed by rearranging the visual features of the letters (Figure 1A), so the letters and the false-font characters were presumed to share similar visual features.
(A) Letters

Digits

Symbols

False-font characters

(B)

$\begin{array}{lc}\text { Word } & \text { BOLD } \\ \text { Nonword } & \text { BILD } \\ \text { Consonants } & \text { BKLD } \\ \begin{array}{l}\text { False-font } \\ \text { characters }\end{array} & \Psi \# \mathbb{O N}\end{array}$

Figure 1. (A) Character sets used in Experiments 1-5. The letters were the 26 uppercase letters from the Microsoft (MS) Sans Serif font. The digits and symbols also were printed in MS Sans Serif. The false-font character set was the same as that used by Maki, Couture, Frigen, and Lien (1997, Experiment 3), who composed the false font by using a font editor to haphazardly rearrange the features found in the upper case letters. (B) Examples of stimuli used by Maki, Couture, et al. (1997, Experiment 3).

Maki, Couture, et al., following the reasoning of Petersen, Fox, Snyder, and Raichle (1990) and Brown, Roos-Gilbert, and Carr (1995), argued that finding an attenuated $\mathrm{AB}$ effect would pinpoint the locus of the interference effect at the semantic, phonological, or orthographic level. Sizeable AB effects were observed in all conditions in which the distractors were composed of letters, but the false-font distractors greatly attenuated the $A B$ effect. Because the blink disappeared when the distractors were composed of false-font characters (i.e., nonletters), Maki, Couture, et al. reasoned that the locus of the interference was at the letter level-that is, below word level, but above the level of individual visual features. They concluded that the confusion among target and distractor letters was responsible for the $\mathrm{AB}$ effect. But what about the AB effect seen with streams of individual letters as distractors (Raymond et al., 1992)? We do not know whether use of the false-font characters (shown in Figure 1A) as distractors will attenuate the AB effect when the targets are single letters. We thus do not know whether a letter distractor $(\mathrm{T} 2+1)$ substitutes for $\mathrm{T} 2$ or whether more elemental visual features of $\mathrm{T} 2$ are partly replaced by those from $\mathrm{T} 2+1$.

From the preceding review, we see that three empirical matters remain to be resolved. First, we suspect, on the basis of intuitions about T-D similarity, that using false-font characters would attenuate the blink when targets are single letters. ${ }^{2}$ But we need an empirical test of that suspicion, in which we would determine the size of 
the $\mathrm{AB}$ effect for the case of single letters as targets and single false-font characters as distractors. Second, T-D similarity has been intuitively determined, so the falsefont characters in Figure 1 appear to be less similar to letters than digits are to letters (for example). But we do not have an empirical verification of this intuition. ${ }^{3}$ Third, the study by Maki, Couture, et al. (1997, Experiment 3 ) fell victim to an unavoidable confounding (that was not noticed at the time). Targets were always words printed in red and green, and distractors were printed in black. Thus, targets were held constant across type of distractor. That practice was necessary in order to distinguish target words from distractor words. But that practice resulted in the targets' differing from false-font strings in two ways: type of font (sans serif vs. false font) and color (red or green vs. black). Thus, the observed attenuation of the $\mathrm{AB}$ effect when the distractors were false-font strings could have been due to the multiple redundant cues distinguishing targets from distractors.

The present series of experiments was intended to provide clarification on the points mentioned above and, thus, to provide a better empirical basis for describing the nature of the interference responsible for the $\mathrm{AB}$ effect. In Experiment 1, we replicated the attenuation of the AB effect by false-font distractors (Maki, Couture, et al., 1997) and generalized the finding to single-character streams. In Experiment 2, we controlled for the number of target features and showed a large $\mathrm{AB}$ effect when targets and distractors were printed in the same color and distractors were false-font characters. In Experiment 3, we measured the properties of the character sets shown in Figure 1, and we showed that, even though false-font characters are neither familiar nor meaningful, mathematical symbols are the set least visually similar to letters. In Experiment 4, using the character sets in Figure 1, we replicated the attenuation of the $\mathrm{AB}$ effect with mathematical symbols as distractors (Chun \& Potter, 1995). In Experiment 5, we demonstrated an asymmetrical T-D similarity effect; in Experiment 4, symbol distractors did not produce much of an $\mathrm{AB}$ effect with letters as targets, but in Experiment 5, letter distractors produced a large $\mathrm{AB}$ effect with symbols as targets. We then will argue from these results that at least part of the interference responsible for the AB effect seems to be at a level lower than that suspected by Maki, Couture, et al. One source of interference appears traceable to the pixel density of distractors and their effectiveness as masking stimuli (Giesbrecht \& Di Lollo, 1998; Grandison et al., 1997; Seiffert $\&$ Di Lollo, 1997). We will close by reconsidering the whole pattern of results in the context of an extended version of the two-stage model of Chun and Potter.

\section{EXPERIMENT 1}

The present experiment was a systematic replication of Experiment 3 from Maki, Couture, et al. (1997). In one part of the experiment, the targets were three-letter words, and the distractors were either three-letter nonwords or three-character false-font strings. In the other part of the experiment, the targets were single letters, and the distractors were either other letters or single falsefont characters. The two targets in each stream were distinguished from the (black) distractors by their presentation in different colors (red and green), following the procedure of Maki, Couture, et al.

\section{Method}

Participants. Texas Tech University undergraduate students $(N=$ 64; 18 male and 46 female), who were enrolled in a general psychology course, volunteered for service in the study and were compensated with course credit. Informed consent was obtained from each participant prior to the beginning of the experimental session.

Apparatus and Materials. The participants were seated in front of microcomputers equipped with color VGA monitors and located in a room illuminated by overhead fluorescent lighting. The letter and false-font character sets displayed in Figure 1 were used in the RSVP lists. Each list consisted of either single characters or strings of three characters. On average, the characters measured approximately $5 \mathrm{~mm}$ wide $\times 6 \mathrm{~mm}$ high. Thus, at the viewing distance used in this experiment (approximately $52 \mathrm{~cm}$ ), the typical character subtended about $0.6^{\circ}$ of visual arc, and a typical three-letter string subtended about $1.8^{\circ}$. All the stimuli were presented in the center of the screen on a light gray background. The targets were presented in either red (T1) or green (T2), and the distractors were presented in black. The luminance of the gray background was $27.3 \mathrm{~cd} / \mathrm{m}^{2}$. The luminance of the black, red, and green letters averaged 24.6, 26.8 , and $32.9 \mathrm{~cd} / \mathrm{m}^{2}$, respectively. The luminance of the false-font characters, averaging $24.7 \mathrm{~cd} / \mathrm{m}^{2}$, was almost identical to that of black letters. ${ }^{4}$ The Experimental Run-Time System (Beringer, 1994) was used to present the visual displays, time all events, and record keyboard responses. All stimulus presentations were synchronized with the monitor's $60-\mathrm{Hz}$ vertical refresh rate.

In the word condition, the targets were 203 consonant-vowelconsonant (CVC) trigrams drawn from the University of South Florida word association norms (Nelson, McEvoy, \& Schreiber, 1998). The distractors were 1,701 nonword CVC trigrams. Eight sets of materials were constructed from the CVC targets and distractors. For each session, 60 pairs of targets (T1 and T2) and surrounding distractors $(\mathrm{T} 1-1, \mathrm{~T} 1+1, \mathrm{~T} 2-1$, and $\mathrm{T} 2+1)$ were randomly selected, subject to the constraints that $\mathrm{T} 1$ and $\mathrm{T} 2$ shared no letters and that $\mathrm{T} 1$ and $\mathrm{T} 2$ shared no letters with their respective surrounding distractors. An additional 300 distractors were randomly selected for each session. In the letter condition, the targets and distractors were single letters, randomly selected on each trial without replacement from the 26-letter alphabet shown in Figure 1.

Design and Procedure. Upon arrival in the laboratory, each of the 64 participants was randomly assigned to one of 64 conditions, distinguished by four between-subjects variables: attentional instructions (2), distractor font (2), order of item type (2), and word set (8). Each participant was given one of two sets of instructions; half of the participants were told to attend to and report the identities of both the first (red) and the second (green) targets, and the other half of the participants were told to ignore the first target and to attend to and report on only the second target. For half of the participants, the distractor items were printed in sans serif font; for the other half of the participants, the distractor items were printed in the false font. During the first half of the experiment, words appeared as targets and distractors for half the participants, and letters appeared in the second half of the experiment; the order was reversed for the other half of the participants. Each of the eight sets of words was assigned to 8 participants. $\operatorname{Lag}(1,2,3,4$, or 6$)$ and type of tar- 
get (words vs. letters) were within-subjects variables. Each lag was presented twice within each block of 10 trials.

After signing the consent form and hearing instructions, each participant experienced 120 RSVP trials, organized into 12 blocks of 10 trials. Six blocks contained words as targets, and 6 blocks contained letters as targets.

Targets and distractors were presented at the rate of $10 / \mathrm{sec}$ (83.3 $\mathrm{msec}$ on, $16.7 \mathrm{msec}$ off ). Each trial began with a fixation stimulus (row of five plus signs) presented for $500 \mathrm{msec}$. A random number of distractors (range: 3-6) preceded T1. A variable number of distractors $(0,1,2,3$, or 5, corresponding to Lags $1,2,3,4$, and 6) preceded T2, and the RSVP list ended with an additional four distractors. Those participants in the two-target condition viewed a prompt to recall $\mathrm{T} 1$, and those participants responded using the keyboard, typing either a letter or a three-letter word. All the participants responded to a prompt to recall T2. Pressing the space bar initiated the next trial.

Data analysis. The first block of trials with each type of target was considered as practice, so the data to be analyzed were obtained from the last five blocks (10 trials at each lag) with each type of stimulus (words vs. letters). The data were averaged over order of stimuli (words-letters vs. letters-words) and over the eight word sets. The data were analyzed separately for words and letters. The result was a $2 \times 2 \times 5$ mixed between-groups within-subjects design with attentional instructions (one vs. two targets) and distractor font (sans serif vs. false font) as between-subjects variables and $\operatorname{Lag}(1,2,3,4$, or 6) as a within-subjects variable. Each cell mean in the design was based on 16 participants contributing results of 10 trials ( 2 trials in each of five blocks).

In the two-target condition, each correct identification of $\mathrm{T} 2$ was counted only if $\mathrm{T} 1$ was identified correctly. Thus, the conditional probability of $\mathrm{T} 2$ given $\mathrm{T} 1$, expressed as a percentage, was the dependent measure for $\mathrm{T} 2$ identification in the two-target instructional condition.

The significance level was set at $p<.05$, the level used throughout this paper.

\section{Results}

The results with three-letter word targets, shown in the left-hand panel of Figure 2, replicated the findings re- ported by Maki, Couture, et al. (1997). A substantial AB effect was found when three-letter words appeared as distractors, but the $\mathrm{AB}$ effect was markedly reduced when the three-character distractor strings contained false-font characters. The right-hand panel of Figure 2 shows that these results generalized to streams containing letter targets and single letters or single false-font characters as distractors.

Identification of words as $\mathbf{T} 2$. The overall $\mathrm{AB}$ effect was significant, as is indicated by the lag $X$ instructions interaction $\left[F(4,240)=9.61, M S_{\mathrm{e}}=149.33\right]$. Moreover, as is shown in Figure 2, the AB effect was modulated by the distractor font; the three-way lag $X$ instructions $X$ distractor font interaction was significant $[F(4,240)=$ $\left.6.99, M S_{\mathrm{e}}=149.33\right]$. All other main effects and interactions were significant [lag, $F(4,240)=38.80, M S_{\mathrm{e}}=$ 149.33 ; instructions, $F(1,60)=52.04, M S_{\mathrm{e}}=705.40$; distractor font, $F(1,60)=144.88, M S_{\mathrm{e}}=705.40$; distractor font $\times$ instructions, $F(1,60)=43.64, M S_{\mathrm{e}}=705.40$; and lag $\times$ distractor font, $F(4,240)=26.33, M S_{\mathrm{e}}=149.33$ ].

The $A B$ effect at each level of distractor font was examined with a two-factor lag $X$ instructions analysis. A reliable $\mathrm{AB}$ effect was obtained when the distractors were letters. The lag $\times$ instructions interaction was significant $\left[F(4,120)=9.59, M S_{\mathrm{e}}=255.75\right]$, as were the main effects of instructions $\left[F(1,30)=49.37, M S_{\mathrm{e}}=\right.$ $1,364.43]$ and lag $\left[F(4,120)=37.51, M S_{\mathrm{e}}=255.75\right]$. Effects of instructions (one vs. two targets) were compared at each lag; all differences were significant [smallest $t(3)=2.32$ ]. No significant $\mathrm{AB}$ effect was found when the distractors were shown in the false font. Performance on T2 varied slightly, albeit significantly, with lag $\left[F(4,120)=3.06, M S_{\mathrm{e}}=42.91\right]$. However, neither the main effect of instructions $\left[F(1,30)=2.81, M S_{\mathrm{e}}=46.37\right]$ nor the lag $\times$ instructions interaction $(F<1)$ was reli-
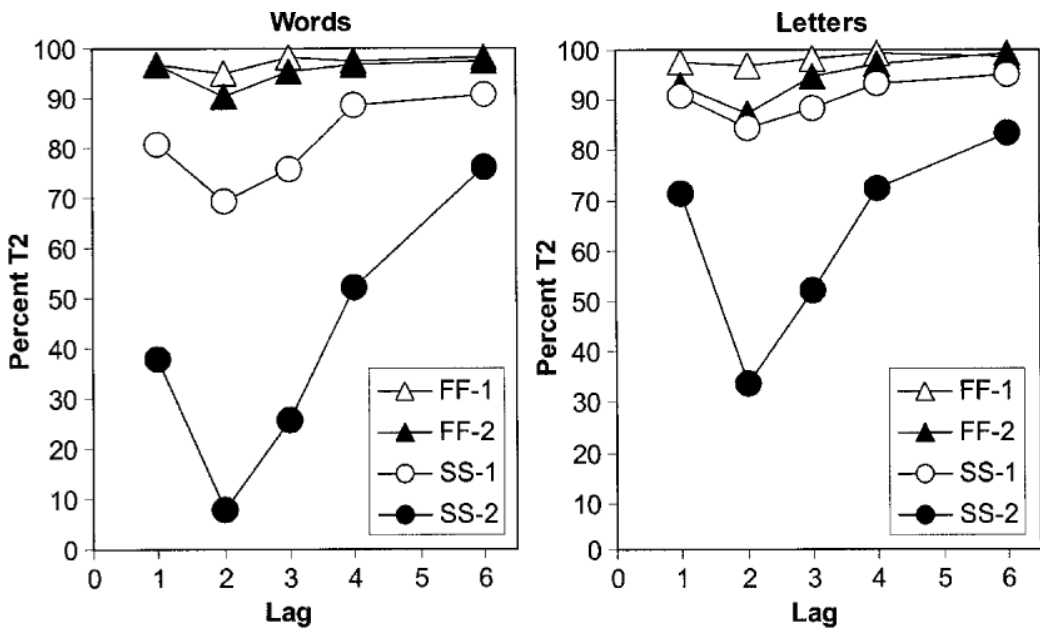

Figure 2. Percentage of second targets correctly identified in Experiment 1. The first target, T1, was printed in red, and the second target, T2, was printed in green. For each type of target (words or letters), data are plotted separately for attentional instructions (one vs. two targets) and distractor font (sans serif [SS] or false font [FF]) as a function of lag (position of $\mathrm{T} 2$ relative to $\mathrm{T} 1$ ). The magnitude of the attentional blink effect is indicated by the difference between the one-target and the two-target functions for each distractor font. 
able. Moreover, none of the comparisons between number of targets at each lag was significant [largest $t(30)=$ 1.49].

Identification of words as T1. In the two-target groups, for Lags 1, 2, 3, 4, and 6, the mean percentages of T1 correctly identified were $64.4 \%, 80.6 \%, 74.4 \%, 76.9 \%$, and $80.6 \%$, respectively, when the distractors were printed in sans serif font. When the distractors contained false-font characters, the corresponding percentages were $77.5 \%, 97.9 \%, 95.0 \%, 96.2 \%$, and $96.9 \%$. Clearly, there was a deficit in $\mathrm{T} 1$ identification at early lags that was reduced when false-font distractors appeared. However, the pattern was different from that shown by T2. A two-factor analysis was performed on the T1 percentages. Both main effects were significant $[F(4,120)=$ $12.18, M S_{\mathrm{e}}=146.27$, and $F(1,30)=32.20, M S_{\mathrm{e}}=364.29$, for lag and distractor font, respectively]. However, the interaction was not significant $(F<1)$.

Identification of letters as $\mathbf{T 2}$. As was the case with words, an overall $\mathrm{AB}$ effect was shown with letters as $\mathrm{T} 2$; the lag $\times$ instructions interaction was significant $\left[F(4,240)=14.44, M S_{\mathrm{e}}=100.72\right]$. Also as was the case with words, the $A B$ effect was attenuated by the use of false-font distractors; the three-way lag $\times$ instructions $\times$ distractor font interaction was significant $[F(4,240)=$ $\left.5.99, M S_{\mathrm{e}}=100.72\right]$. All other main effects and interactions were significant $\left[\operatorname{lag}, F(4,240)=33.08, M S_{\mathrm{e}}=\right.$ 100.72 ; instructions, $F(1,60)=34.99, M S_{\mathrm{e}}=569.65$; font, $F(1,60)=55.09, M S_{\mathrm{e}}=569.65$; distractor font $\times$ instructions, $F(1,60)=19.99, M S_{\mathrm{e}}=569.65$; and lag $\times$ distractor font, $\left.F(4,240)=14.29, M S_{\mathrm{e}}=100.72\right]$.

Separate two-factor (lag $\times$ instructions) analyses were used to examine the $A B$ effect when different fonts were used to display distractors. The AB effect was significant when the distractors were letters. The lag $\times$ instructions interaction was reliable $[F(4,120)=11.81$, $\left.M S_{\mathrm{e}}=163.21\right]$, as were the two main effects of instructions $\left[F(1,30)=29.14, M S_{\mathrm{e}}=1,054.58\right]$ and $\operatorname{lag}[F(4,120)=$ $\left.27.54, M S_{\mathrm{e}}=163.21\right]$. The differences between attentional instructions (one vs. two targets) at each lag were significant [smallest $t(30)=2.70$ ]. When the distractors were displayed as false fonts, the $\mathrm{AB}$ effect nearly disappeared but was reliable, as was indicated by a significant lag $\times$ instructions interaction $[F(4,120)=3.18$, $\left.M S_{\mathrm{e}}=37.14\right]$. The small main effects were also reliable [for instructions, $F(1,30)=7.01, M S_{\mathrm{e}}=84.72$; for lag, $\left.F(4,120)=6.77, M S_{\mathrm{e}}=37.14\right]$. The differences between attentional instructions favored one-target over two-target instructions and tended to be significant at the shortest lags $[t(30)=1.92, p<.10$, for Lag 1 , and $t(30)=2.94$, for Lag 2]; none of the other comparisons was significant [largest $t(30)=1.52$ ]. Thus, there were signs of an $\mathrm{AB}$ effect with letter targets when false-font distractors were used, but, as is clear from Figure 2, the effect was much smaller than the $\mathrm{AB}$ effect observed with letter distractors.

Identification of letters as T1. As with word targets, identification of T1 with letter distractors was less accurate than identification of $\mathrm{T} 1$ with false-font distractors.
In the two-target groups, for Lags 1, 2, 3, 4, and 6, the mean percentages correct for $\mathrm{T} 1$ were $78.8 \%, 83.8 \%$, $83.1 \%, 80.0 \%$, and $80.0 \%$ for letter distractors; the corresponding averages for false-font distractors were $86.9 \%$, $95.6 \%, 97.5 \%, 96.2 \%$, and $96.9 \%$. The main effects of both lag $\left[F(4,120)=3.31, M S_{\mathrm{e}}=85.19\right]$ and distractor font $\left[F(1,30)=14.75, M S_{\mathrm{e}}=494.25\right]$ were significant, but the interaction was not reliable $\left[F(4,120)=1.20, M S_{\mathrm{e}}=\right.$ 85.19]. As with word targets, the pattern of deficits with $\mathrm{T} 1$ identification did not track the pattern seen for T2.

\section{Discussion}

There are two principal findings in this experiment that will serve as baselines for the experiments to follow. First, the present results replicated the findings of Maki, Couture, et al. (1997). An AB effect was observed when the targets were words and the distractors were nonwords. The AB effect was considerably reduced when the distractors appeared printed in the false font. Second, a parallel set of results was found when the targets and the distractors were single characters. An AB effect was obtained when the targets were letters and the distractors were also letters. However, the AB effect was diminished when single false-font characters appeared as distractors.

\section{EXPERIMENT 2}

In the preceding experiment (and in Maki, Couture, et al., 1997, Experiment 3), two features distinguished targets from distractors: font and color. Thus, the attenuation of the $\mathrm{AB}$ effect seen with false-font distractors could be due to the number of distinctive target features, rather than to the distinctiveness of the false-font characters (as determined in Experiment 1). The purpose of this experiment was to reduce the number of target features by presenting targets and distractors in one color (black). If we still obtained an attenuation of the blink with falsefont distractors, familiarity and/or meaningfulness of distractors would be implicated as the principal determiners of T-D similarity effects. But if an AB effect were to be found with false-font distractors, other dimensions of T-D similarity must be considered as possible sources of interference responsible for the $\mathrm{AB}$.

\section{Method}

Participants. Undergraduate students, 23 female and 9 male, were obtained from the same pool as that in Experiment 1. These participants were randomly assigned in equal numbers to the betweengroups conditions described below.

Design and Procedure. This experiment was conducted exactly like Experiment 1, with the following few exceptions. All the targets were displayed in black. The participants saw streams consisting of three-character or one-character strings, but all the distractors were displayed in the false font (Figure 1). Those participants instructed to watch for two targets (T1 and T2) saw two 3-letter words as targets or two single letters as targets, both printed in the sans serif font (Figure 1). For those participants instructed to report on one target, only T2 was present in the RSVP list, and T1 was replaced by a distractor. Each of the eight sets of words was assigned to 4 participants; word targets occurred first for half the participants, and letter targets occurred first for the other half of the par- 
ticipants. As in Experiment 1, neither word set nor word-letter order was included as a factor in the analysis. The resulting design for each type of target (words vs. letters) was a $2 \times 5$ factorial, with attentional instructions (one vs. two targets) as the between-groups variable and lag $(1,2,3,4$, or 6$)$ as the within-subjects variable.

\section{Results}

In this experiment, and in contrast to the results of Experiment 1 , a substantial $\mathrm{AB}$ effect was found with falsefont distractors. Figure 3 shows the effect for word targets (left-hand panel) and for single-letter targets (right-hand panel).

Identification of words as T2. As in Experiment 1, the data were analyzed separately for word and letter targets. T2 identification in the two-target condition, also as in Experiment 1, was scored contingently on correct identification of $\mathrm{T} 1$. The effects of both instructions and lag were significant $\left[F(1,30)=32.00, M S_{\mathrm{e}}=420.83\right.$, and $F(4,120)=16.87, M S_{\mathrm{e}}=164.02$, respectively $]$. The AB effect in this experiment was manifested as a significant lag $\times$ instructions interaction $\left[F(4,120)=23.93, M S_{\mathrm{e}}=\right.$ 164.02]. The interaction was explored by comparing the one- and two-target instructional conditions at each lag. The differences, all showing lower accuracy in the twotarget condition, were significant at Lags 2-4 [smallest $t(30)=2.42$ ]. The differences at Lags 1 and 6 were not significant (both $t \mathrm{~s}<1$ ).

Identification of words as T1. For the two-target group, the average percentages of $\mathrm{T} 1$ words correctly identified were $69.4 \%, 93.8 \%, 90.0 \%, 93.8 \%$, and $93.1 \%$ for Lags $1-6$, respectively. The significant effect of lag $\left[F(4,60)=14.40, M S_{\mathrm{e}}=123.12\right]$ reflects the lower accuracy at Lag 1.

Identification of letters as T2. Letter targets also showed an $\mathrm{AB}$ effect. Both main effects were significant
$\left[F(1,30)=4.88, M S_{\mathrm{e}}=811.36\right.$, and $F(4,120)=4.70$, $M S_{\mathrm{e}}=235.35$, for instructions and lag, respectively]. The instructions $\times$ lag interaction was also significant $\left[F(4,120)=6.33, M S_{\mathrm{e}}=235.35\right]$. T2 accuracy was lower for the two-target instructions at Lags 2 and $4[t(30)=$ 3.24 and $t(30)=2.50]$ and also tended to be lower at Lag $3[t(30)=1.79, p<.10]$. The effect of instructions was not significant at Lag $6[t(30)=1.40]$. The reverse effect at Lag 1, where performance was actually better with two-target instructions than with one-target instructions, was reliable $[t(30)=2.36]$.

Identification of letters as T1. For the two-target group, the mean percentages of T1 words correctly recalled were $66.2 \%, 80.0 \%, 81.2 \%, 80.6 \%$, and $78.1 \%$ for Lags 1, 2, 3, 4, and 6, respectively. The significant effect of lag $\left[F(4,60)=3.20, M S_{\mathrm{e}}=196.21\right]$ results from the lower accuracy at Lag 1.

\section{Discussion}

In this experiment, we found a large $\mathrm{AB}$ effect for both word and letter targets when only one feature (font) distinguished the targets from the distractors. The AB effect was attenuated by false-font distractors only when the targets and the distractors differed on an additional dimension (color) in Experiment 2 (and in Experiment 3 of Maki, Couture, et al., 1997). Therefore, although falsefont characters are certainly less familiar and less meaningful than are letters (as we shall show in Experiment 3), familiarity and meaningfulness by themselves cannot account for the pattern of the results accumulated thus far.

The results also revealed a difference between words and letters with respect to their sensitivity to interference from false-font distractors. This difference was manifested in two ways. First, when both targets were included in
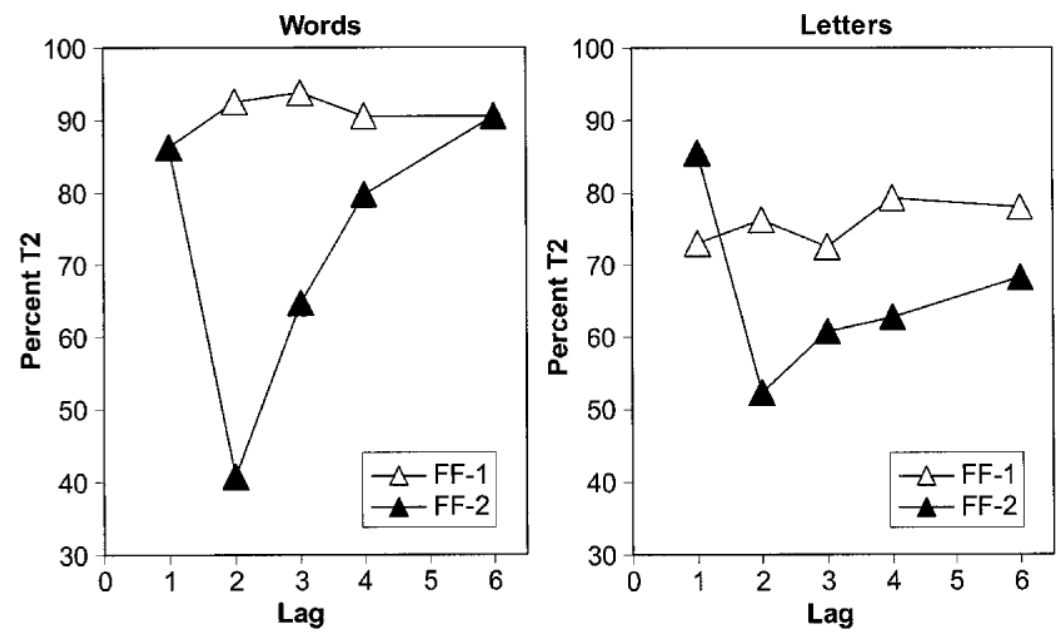

Figure 3. Percentage of second targets (T2) correctly identified in Experiment 2. The targets were either words or letters, and the distractors were always displayed in the false font (FF). Unlike in Experiment 2, the targets were printed in black. Data are plotted separately for the two attentional instructions (one vs. two targets) as a function of T1-T2 lag. The magnitude of the attentional blink effect is indicated by the difference between the one-target and the two-target functions for each distractor font. 
the RSVP streams, reporting of T1 was less accurate for letters than for words. Second, when only T2 appeared, reporting of $\mathrm{T} 2$ was less accurate for letters than for words. One way of interpreting these effects would be to cite the lexical and semantic properties of words that are not possessed by letters (e.g., as in the interactive activation model of McClelland \& Rummelhart, 1981). Words might thus be more easily discriminated from false-font strings than are letters (at least when the number of target features is controlled). Thus, detecting one letter in a stream of false-font characters would be rather difficult, resulting in poor performance on $\mathrm{T} 1$ and on $\mathrm{T} 2$ when only $\mathrm{T} 2$ had to be reported. But when both targets had to be reported, detecting one target might have acted as a preparatory signal enabling easier detection and report of the target that immediately followed. In this case, reporting T3 following successful detection of $\mathrm{T} 1$ would be easier than reporting T2 alone (as in the right panel of Figure 3).

\section{EXPERIMENT 3}

Chun and Potter (1995) found that the AB effect with letter targets was substantially smaller when mathematical symbols were distractors than when digits were distractors. Chun and Potter observed that the interpretation of this difference was complicated by covariation between conceptual and visual properties distinguishing the various character sets. They observed (but without direct measurement) that the letters were more visually distinct from symbols than from digits. They also observed that the names of the symbols were less familiar than those of digits and letters. They called for additional research to "clarify and separate the effects of visual similarity and categorical similarity" (p. 122). The present study was intended to do just that by measuring conceptual variables (meaningfulness and familiarity) and visual variables (featural similarity and pictorial density) for each of the character sets shown in Figure 1A. By obtaining direct measurements of the properties of the stimulus sets shown in Figure 1A, we hoped to identify the sources of T-D similarity that would be candidates for organizing the results of Chun and Potter and of Maki, Couture, et al. (1997; see note 3 ). We recruited college students from the same population as that used in the RSVP studies (Experiments 1-2). The students rated the 68 characters in Figure 1A on familiarity and meaningfulness and enumerated the visual features of each of those characters. We also measured the dot-level density of each character by counting the number of picture elements (pixels). We then compared each character with every other character in order to compute the visual similarity on the basis of both visual features and pixel density.

\section{Method}

Participants. Undergraduate students were recruited from the same population as that in Experiment $1(N=56 ; 19$ male and 37 female). The participants were randomly assigned (in equal numbers) to the four groups described below.
Materials. The stimuli consisted of 26 uppercase letters, eight digits, and eight mathematical symbols printed in the MS Sans Serif font; the digits and symbols were those identified by Chun and Potter (1995). Also included were the 26 false-font characters used by Maki, Couture, et al. (1997, Experiment 3). All four character sets are shown in Figure 1A.

The characters in each set were randomly divided into two subsets, each consisting of 13 letters, four digits, four symbols, and 13 false-font characters. These 34-character subsets will be referred to as Set 1 and Set 2 . The characters in each set were randomly ordered and printed on a single page with instructions to rate the characters with respect to familiarity. The rating alternatives were very familiar-I see it several times each day, somewhat familiar-I see it occasionally, not very familiar-I think I've seen it somewhere, and not at all familiar-I'm sure I've never seen it. The same order of characters appeared on a second page with instructions to rate the characters with respect to meaningfulness, where meaningfulness was defined in terms of the ease with which the character could be named. The rating alternatives for meaningfulness were very meaningful_I know the name very well, somewhat meaningful-I have to think about the name, not very meaningful-I think I may be able to name it, and not at all meaningful-I'm sure I don't know its name. The participants entered their ratings on optical scanning sheets.

For purposes of recording the participant's identification of visual features, the letters, digits, and mathematical symbols in each set were randomly ordered and listed on another form, followed by the false-font characters in that set, also randomly ordered. A row of eight empty squares appeared next to each character, in which the participants sketched visual features associated with the character.

The familiarity and meaningfulness rating pages from one set were combined with the visual feature identification pages from the other set, yielding two booklets. Thus, one booklet contained ratings for the characters in Set 1 and the visual feature identification task for characters in Set 2 . The other booklet contained ratings for the characters in Set 2 and the visual feature identification task for Set 1 .

Design and Procedure. The participants were tested in four groups, with each booklet being used for each of two groups. The participants began by rating familiarity and meaningfulness. Then they were given instructions on decomposing the characters into component features. They were told to assume that they needed to communicate with a visitor from another planet ("Mr. Spock from the planet Vulcan") about our written language and that they needed to describe to the visitor the basic visual features of some written characters. The experimenter demonstrated the technique of identifying and sketching the visual features, working with two sample characters created for the purposes of these instructions. The experimenter then demonstrated extraction of simple features (lines and arcs) and complex features (vertices). The participants were told that they should try to minimize the number of features they created but to sketch enough features that the character could be completely reconstructed on the basis of their list of features. The participants were also told to respect the location of the features by sketching their features in the appropriate location in the boxes. Using the sample characters, the participants were shown how to reuse features across characters. After the entire group of participants had finished identifying the visual features for the list of letters, digits, and symbols, they were reinstructed regarding the falsefont characters. They were told that the task was to pretend that they were the visitor from space and that they needed to describe their own written language by identifying visual features for the characters in that language. The participants then performed the visual feature identification task on the false fonts.

Data analyses. Both familiarity and meaningfulness were scored on a 4-point scale, with 3.00 being most familiar or most meaningful and 0.00 being least familiar or least meaningful.

The visual characteristics of each of the four sets of characters shown in Figure 1A were examined at two levels_-visual features 
and pixels. For each character, we determined the average number of features sketched for that character. To determine the dissimilarities among character sets, we began by counting the number of times a visual feature was sketched by the participants for each character. Visual features that were nominated by only 1 participant were eliminated from the subsequent analyses. The result was a frequency table defined by 68 rows (the characters from Figure 1A) and 121 columns (the remaining features). The chi-square statistic, as a measure of dissimilarity, was computed for each pair of characters. Then the chi-square values were averaged to obtain mean dissimilarity scores for each letter with respect to each category of character. That is, the chi-square values for each of the 26 letters were averaged over all other letters, all digits, all symbols, and all false-font characters. For example, the letter A received four scores: its average dissimilarity relative to all other letters, its average dissimilarity relative to all digits, its average dissimilarity relative to all symbols, and its average dissimilarity relative to all false-font characters. The dissimilarity analyses focused on the dissimilarity between letters and other character sets, because letters are typically used as targets and other character sets appear as distractors (e.g., Chun \& Potter, 1995; Maki, Couture, et al., 1997).

A font editor was used to display each of the characters shown in Figure 1A. Enlarged images were created, and the number of pixels was computed for each character. To compute dissimilarities among character sets on the basis of pixel frequencies, we computed the difference between the pixel frequencies for each pair of characters. A root mean-squared difference (i.e., standard deviation) was computed for each character set for each letter. Thus, each letter received four scores reflecting its average dissimilarity to each of the four character sets (remaining letters, all digits, all symbols, and all false-font characters).

Usable results were obtained from 53 participants for the familiarity and meaningfulness ratings. (The other participants made errors or omissions in marking the optical scanning sheets.) The scores on these ratings were entered into an analysis of variance using error terms based on participants. All the measures were also analyzed using error terms based on items. For the rating and frequency measures, the between-groups analyses of variance among items were based on four groups of characters (26 letters, eight digits, eight symbols, and 26 false-font characters). Within-groups analyses of variance were used for the dissimilarity analyses, in which the dissimilarities for the 26 letters were contrasted among the four character sets. Post hoc $t$ tests were conducted to assess significance of differences between pairs of means, with Bonferroni adjustments for multiple comparisons.

\section{Results}

The mean ratings are displayed in Table 1 for all four types of characters. The table shows that false-font char- acters were rated both low in familiarity and low in meaningfulness. The mathematical symbols, although rated high in both familiarity and meaningfulness, tended to be rated slightly lower than both the letters and the digits. These results were true of both booklets, so booklet was not included as a factor in the analyses.

The average number of visual features did not distinguish the character sets. However, the symbol set was distinguished from the other three sets of characters on the bases of feature dissimilarity and both pixel frequency and pixel dissimilarity.

Familiarity. The effect of stimulus type was reliable [for participants, $F(3,156)=1,355.99, M S_{\mathrm{e}}=0.054$; for items, $\left.F(3,64)=177.97, M S_{\mathrm{e}}=0.169\right]$. The post hoc analyses using participants showed that the rated familiarity of letters and digits did not differ $(p<.10)$ but that all other comparisons were significant. The post hoc analyses using items indicated that the false-font characters were rated significantly less familiar than the other three character sets (which did not differ among themselves). In both analyses, the contrast between the false-font set and the other three sets accounted for over $99 \%$ of the variance among sets. Thus, false-font characters were rated reliably less familiar than any of the other stimulus types.

Meaningfulness. The effect of stimulus type was significant for both participants $\left[F(3,156)=1,766.32, M S_{\mathrm{e}}=\right.$ $0.046]$ and items $\left[F(3,64)=181.92, M S_{\mathrm{e}}=0.183\right]$. The post hoc analyses showed a pattern identical to that shown in the familiarity ratings. False-font characters were rated as significantly less meaningful than any of the other three stimulus types in both analyses using participants and items. In the analysis using participants, symbols were rated as less meaningful than either letters or digits. In both analyses, as was the case for familiarity, the contrast between the false-font set and the other three sets combined accounted for over $99 \%$ of the variance among stimulus types. Thus, the principal effect in the meaningfulness ratings was that false-font characters were viewed as less meaningful than any of the other three stimulus types.

Feature density. The number of visual features sketched for each character was computed for each participant and then averaged to obtain the mean number of features assigned to each individual character. These

Table 1

Stimulus Characteristics of Letters, Digits, Symbols, and False-Font Characters

\begin{tabular}{|c|c|c|c|c|c|c|c|c|}
\hline \multirow[b]{2}{*}{ Measure } & \multicolumn{2}{|c|}{ Letters } & \multicolumn{2}{|c|}{ Digits } & \multicolumn{2}{|c|}{ Symbols } & \multicolumn{2}{|c|}{ False Font } \\
\hline & $M$ & $S E$ & $M$ & $S E$ & $M$ & $S E$ & $M$ & $S E$ \\
\hline Familiarity & 2.97 & 0.013 & 3.00 & 0.000 & 2.76 & 0.045 & $0.56 *$ & 0.046 \\
\hline Meaningfulness & 2.97 & 0.017 & 2.99 & 0.014 & 2.85 & 0.036 & $0.45 *$ & 0.045 \\
\hline Feature density & 2.46 & 0.121 & 2.36 & 0.133 & 2.59 & 0.407 & 2.56 & 0.122 \\
\hline Feature dissimilarity & 10.65 & 0.143 & 10.62 & 0.174 & $11.00^{*}$ & 0.164 & 10.55 & 0.181 \\
\hline Pixel density & 67.73 & 3.38 & 59.12 & 2.63 & $38.00^{*}$ & 4.81 & 62.69 & 4.25 \\
\hline Pixel dissimilarity & 22.89 & 1.39 & 18.07 & 1.81 & $33.97 *$ & 2.66 & 26.95 & 1.20 \\
\hline
\end{tabular}

Note-Familiarity and meaningfulness data are average ratings based on 53 participants. For familiarity and meaningfulness, the maximum rating was 3.0 and the minimum rating was 0.0 . The other four measures are based on 26 letters, eight digits, eight symbols, and 26 false-font characters. (The stimulus sets are shown in Figure 1.) Means marked with an asterisk differ significantly from the means of the other three sets of characters on that measure. See the text for an explanation of the dissimilarity measures. 
means were averaged for each character set to produce the means shown in Table 1. The four character sets did not differ significantly, as is indicated by the nonsignificant effect of stimulus type $(F<1)$.

Feature dissimilarity. The mean dissimilarity scores (and standard errors) shown in Table 1 differed significantly $\left[F(3,75)=7.96, M S_{\mathrm{e}}=0.132\right]$. The post hoc analyses of pairwise comparisons among means showed that the average dissimilarity between letters and symbols was significantly higher than the other three average dissimilarity scores $(p \mathrm{~s}<.01)$. The average dissimilarities between letters and other letters, digits, and false-font characters were not significantly different. The contrast between the symbols and the other three sets combined accounted for $96 \%$ of the variance among character sets. Thus, in contrast to the familiarity and meaningfulness ratings, this analysis of dissimilarities among the character sets on the basis of visual features shows that the symbol set is most distinctive.

Pixel density. The mean numbers of pixels per character in each character set are shown in Table 1 . The character sets differed significantly $[F(3,64)=5.63$, $\left.M S_{\mathrm{e}}=325.84\right]$. The post hoc analyses showed that symbols contained significantly fewer pixels than did any other character set. The digit and symbol sets were significantly different only when the Bonferroni adjustment was not made. Otherwise, letters, digits, and false-font characters did not differ significantly in terms of their average numbers of pixels. The contrast between the symbols and the other three character sets combined accounted for $93 \%$ of the variance among character sets.

Pixel dissimilarity. The average dissimilarity scores based on pixel densities, shown in the last row of Table 1, differed significantly $\left[F(3,75)=23.76, M S_{\mathrm{e}}=49.56\right]$. The post hoc analyses showed that the symbol set was significantly different from letters and digits and marginally significantly different from the false-font set $(p<$ .06). Moreover, the means of the letter, digit, and falsefont character sets were all significantly different from one another. The contrast between the symbols and the other three sets accounted for $71 \%$ of the variance among the sets of characters.

\section{Discussion}

The results of this experiment (Table 1) show that the false-font characters were regarded by our participants as not familiar and not meaningful and, hence, exceptional relative to the other three sets (letters, digits, and mathematical symbols). Equally diagnostic were the feature dissimilarity, pixel density, and pixel dissimilarity measures. But these three measures marked the symbol set as exceptional. Thus, the symbol set was less dense in pixels and was more visually distinctive relative to the other three sets. It remained to acquire experimental evidence, as we did in Experiments 4 and 5, that would bear on the relative importance of two visual characteristics (visual features and pixel density) as sources of T-D similarity.

\section{EXPERIMENT 4}

The purpose of this experiment was to replicate Chun and Potter's (1995) results with single letters as targets and single symbols or digits as distractors. A successful replication would place Chun and Potter's results in the context of the present set of experiments, using stimuli with empirically determined properties (Table 1).

\section{Method}

Participants. Another 32 undergraduate students (19 female, 13 male) were recruited from the same population as that in the previous experiments and were randomly assigned in equal numbers to the four groups mentioned below.

Procedure. In respects not mentioned here (such as the apparatus, stimuli, and timing), the methods were identical to those for letter targets in Experiment 3. The targets were all uppercase letters other than I, O, Q, and Z. The distractors were randomly drawn from the sets of digits or symbols shown in Figure 1, with the constraint that a distractor could not occur twice in succession. The trials were organized into 5-trial randomized blocks (1 for each lag). Each participant experienced 22 such blocks (110 trials) with one distractor set and then 22 more blocks with the other distractor set. For each type of distractor, the first 10 trials were for practice, and data from the next 100 trials were used in the analyses, resulting in 20 trials per lag. Four groups of participants were distinguished by the type of instructions (one vs. two targets) and order of distractor set (digits first vs. symbols first). As in the previous experiments, the order of distractor sets was ignored in the analyses, resulting in a $2 \times 5 \times 2$ mixed design with distractor and lag as within-subjects variables and instructions as a between-groups variable.

\section{Results}

The principal finding in this experiment, in accord with that of Chun and Potter (1995), was a large AB effect when the task was to search for a letter among digit distractors but a much smaller $\mathrm{AB}$ effect when the task was to search for a letter among symbol distractors. Figure $4 \mathrm{~A}$ shows the mean percentages of trials in which $\mathrm{T} 2$ was correctly identified; the means are displayed separately for distractor (digits vs. symbols), instructions (one vs. two targets), and lag (1, 2, 3, 4, or 6). For the digit distractors, accuracy for the two-target instructions dropped from about $90 \%$ at Lag 1 to $65 \%-70 \%$ at Lags 2 and 3 and recovered somewhat at Lags 4 and 6 . The corresponding drop in accuracy at Lags 2 and 3 when symbols were the distractors was only to about $85 \%$.

Identification of T2. A three-factor analysis of variance was performed on the percentages of T2 identification, including instructions (one target vs. two targets) and lag $(1,2,3,4$, or 6$)$ as within-subjects variables and distractor (digits vs. symbols) as a between-groups variable. The overall AB effect was indicated by the significant interaction of instructions and $\operatorname{lag}[F(4,120)=8.64$, $\left.M S_{\mathrm{e}}=76.79\right]$. But the size of the $\mathrm{AB}$ effect, as is shown in Figure 4, depended on the type of distractor, resulting in the significant three-way interaction $[F(4,120)=4.39$, $\left.M S_{\mathrm{e}}=44.47\right]$. All other main effects and interactions were significant [smallest $F(4,120)=7.24, M S_{\mathrm{e}}=44.47$ ] 

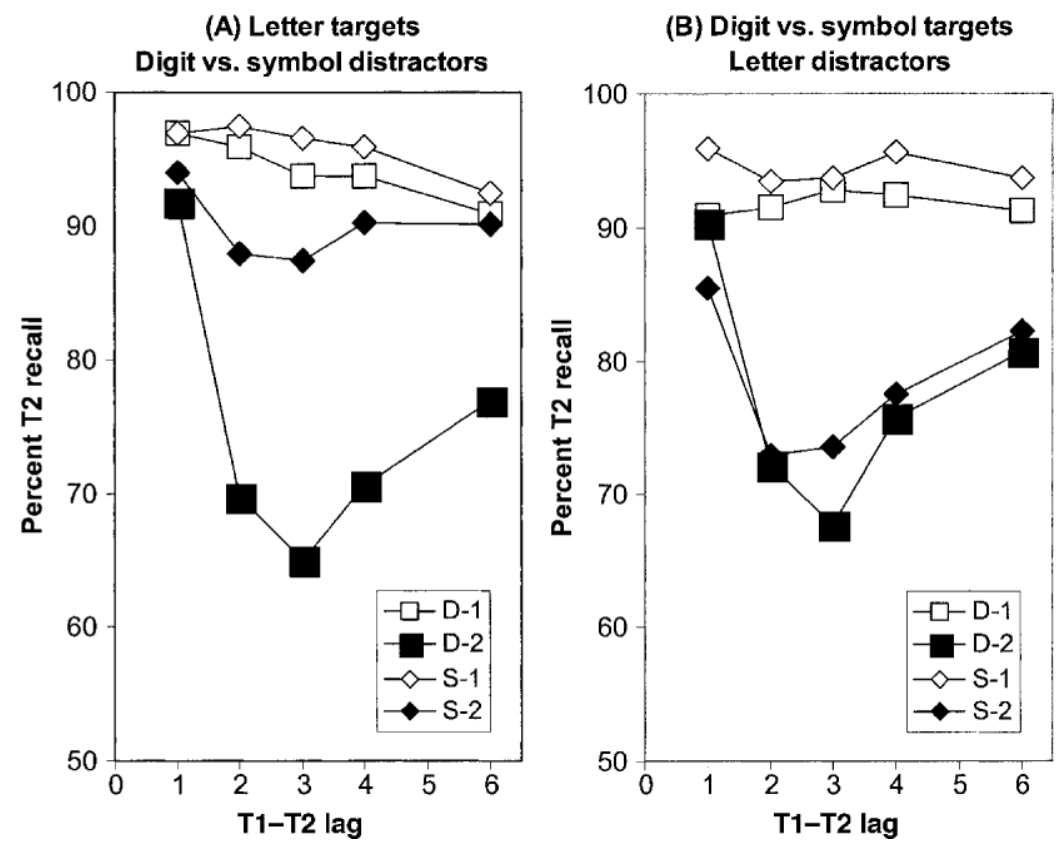

Figure 4. Percentages of second targets (T2) correctly identified in Experiments 4 and 5. Data are plotted separately for the two attentional instructions (one vs. two targets) as a function of T1-T2 lag. In Experiment 4 (A, left panel), the targets were letters, and the distractors were either the digits $(D)$ or the symbols $(S)$ shown in Figure 1. The magnitude of the attentional blink effect is indicated by the difference between the one-target and the two-target functions for each type of distractor. In Experiment 5 (B, right panel), the target and distractor sets were the inverse of those used in Experiment 4. The targets were either the digits (D) or the symbols (S) shown in Figure 1 , and the distractors were the letters. The magnitude of the attentional blink effect is indicated by the difference between the one-target and two-target functions for each type of target.

Separate two-way analyses of variance performed for each type of distractor included lag and instructions as factors. For symbol distractors, only the effect of instructions was significant $\left[F(1,30)=12.44, M S_{\mathrm{e}}=111.97\right]$, indicating that performance was worst with two targets [smallest other $\left.F(4,120)=1.95, M S_{\mathrm{e}}=46.51\right]$. For digit distractors, performance with two targets was also worse than performance with a single target $[F(1,30)=45.51$, $\left.M S_{\mathrm{e}}=332.21\right]$, but there was also a significant effect of $\operatorname{lag}\left[F(4,120)=14.08, M S_{\mathrm{e}}=74.75\right]$ and a significant instructions $\times$ lag interaction $\left[F(4,120)=10.28, M S_{\mathrm{e}}=\right.$ 74.75].

The effects of lag with digit distractors were examined separately for the one- and two-target conditions. The overall effects of lag were significant for both target conditions [for one target, $F(4,60)=4.01, M S_{\mathrm{e}}=21.18$; for two targets, $\left.F(4,60)=13.53, M S_{\mathrm{e}}=128.32\right]$. A trend analysis revealed a linear component for both target conditions $\left[F(1,15)=23.03, M S_{\mathrm{e}}=13.74\right.$, and $F(1,15)=$ $10.76, M S_{\mathrm{e}}=121.74$, for one and two targets, respectively]. However, the quadratic component was significant for the two-target condition $\left[F(1,15)=19.44, M S_{\mathrm{e}}=\right.$ 267.22], but not for the one-target condition $(F<1)$. These results, along with the display in Figure 4A, indi- cate that a pattern of data consistent with the $\mathrm{AB}$ effect was most in evidence with digit distractors.

Identification of T1. The percentages of first targets correctly identified were available from the groups given two-target instructions. For the digit distractors, the percentages were $89.1 \%, 93.8 \%, 92.8 \%, 94.4 \%$, and $93.1 \%$ for Lags 1, 2, 3, 4, and 6; for the symbol distractors, the percentages were $89.7 \%, 95.6 \%, 96.9 \%, 97.8 \%$, and 95.6\% for Lags 1, 2, 3, 4, and 6, respectively. The somewhat lower performance at Lag 1 resulted in a significant effect of lag $\left[F(4,120)=216.17, M S_{\mathrm{e}}=32.31\right]$. The effect of distractor was not significant $\left[F(1,30)=2.83, M S_{\mathrm{e}}=\right.$ $88.25]$, and the interaction was not significant either $(F<1)$.

\section{Discussion}

In this experiment, we successfully replicated Chun and Potter (1995) in showing that a large AB effect was present when the task was to report two letters from a stream of digits but that the $\mathrm{AB}$ effect was much smaller when the task was to report two letters from a stream of mathematical symbols. The effects obtained here (Figure 4A) matched the effects reported by Chun and Potter (1995, Figure 7) quantitatively as well as qualitatively and, thus, served as a baseline for the next experiment. 


\section{EXPERIMENT 5}

Finding the attenuation of the $\mathrm{AB}$ effect with symbol distractors in the preceding experiment connected the $\mathrm{AB}$ effect to the last three rows of Table 1. Symbols were distinguished from the other three sets of characters in Experiment 3 with respect to dissimilarity based on visual features and dissimilarity based on pixel density. It remained to decide between these two sources of interference as to their relative contributions to the $A B$ effect. One possibility is that visual features in targets are overwritten by visual features in subsequent distractors. Another possibility is that parts of targets are obscured by the dot patterns present in the following distractors (Giesbrecht \& Di Lollo, 1998; Grandison et al., 1997; Seiffert \& Di Lollo, 1997). The latter suggestion implies that more dense distractors will function as more effective masks of preceding targets. Thus, in Experiment 4, digits interfered with the processing of letters more so than did symbols, resulting in a larger AB effect for digits than for symbols. However, an asymmetry is predicted by the dot-level masking account. Letters, being relatively more dense than symbols, should mask both digits and symbols when digits and symbols are targets and letters are distractors. ${ }^{5}$ Thus, the dot-level masking view predicts substantial $A B$ effects both when digits are targets among letters and when symbols are targets among letters.

\section{Method}

Participants. The participants were recruited from the same population as that in Experiment 4 and included 22 females and 10 males.

Procedure. The experiment was conducted exactly like Experiment 4, with the following few exceptions. Most important, the functions of the target and the distractor sets were switched in this experiment. The uppercase letters from Experiment 4 became distractors in this experiment, and the two distractor sets (digits and symbols) from Experiment 4 became two target sets in this experiment. The keys corresponding to the digits 2-9 on the keyboard were covered with opaque stickers printed with the numbers and symbols shown in Figure 1 (" $2<$, , "3>,", "4=," . . . " "9*"). The participants were instructed to use these keys to identify both types of targets. As in Experiment 4, the design was a $2 \times 5 \times 2$ mixed design with target (digits vs. symbols) and lag (1, 2, 3, 4, or 6) as within-subjects variables and instructions (one vs. two targets) as the betweengroups variable.

\section{Results}

This experiment produced a strong $\mathrm{AB}$ for the reversed task of searching for digit targets in a string of letters. But, as is shown in Figure 4B, an equally large AB effect was obtained when the task was to search for symbol targets in a stream of letters. Contrary to Experiment 4, there was no significant difference between digits or symbols and the magnitude of the blink they produced.

Identification of T2. Searching for two targets produced lower performance than did searching for only one target, as was indicated by the significant main effect of instructions $\left[F(1,30)=43.74, M S_{\mathrm{e}}=427.64\right]$. Also, performance varied significantly as a function of $\operatorname{lag}[F(4,120)=$
8.83, $\left.M S_{\mathrm{e}}=77.88\right]$. The AB effect shown in Figure 4B was reliable, as was indicated by the significant instructions $\times$ lag interaction $\left[F(4,120)=8.49, M S_{\mathrm{e}}=77.88\right]$. However, Figure 4B shows little variation in the AB effect that was due to type of target, and this was statistically confirmed by the lack of any effects of target [largest $\left.F(1,30)=1.97, M S_{\mathrm{e}}=138.07\right]$. In particular, the threeway interaction was not significant $\left[F(4,120)=1.46, M S_{\mathrm{e}}=\right.$ 77.88], confirming that the instructions $\times$ lag interaction that is the signature of the AB effect was not modulated by the type of target.

Identification of T1. Percentages of trials on which a digit $\mathrm{T} 1$ was correctly identified (by those participants receiving two-target instructions) were $82.2 \%, 86.2 \%$, $90.0 \%, 91.2 \%$, and $92.5 \%$ for Lags 1, 2, 3, 4, and 6, respectively. Corresponding percentages for symbol T1 trials were $83.4 \%, 91.2 \%, 96.2 \%, 94.7 \%$, and $93.4 \%$. There was a reliable effect of $\operatorname{lag}\left[F(4,60)=16.19, M S_{\mathrm{e}}=\right.$ 46.07] that resulted mostly from lower performance at Lag 1. The slight tendency for T1 performance to be more accurate for symbol targets than for digit targets did not achieve significance $\left[F(1,15)=3.40, M S_{\mathrm{e}}=\right.$ 133.96]. Importantly, the target $\times$ lag interaction was not reliable $(F<1)$, indicating that the lag effect on $\mathrm{T} 1$ performance was not modulated by the type of target.

\section{Discussion}

The characters in the target and distractor sets of Experiment 4 (and from Chun \& Potter, 1995) were switched in this experiment. Letters were used as distractors, and digits and symbols became the targets. As is shown in Figure $4 \mathrm{~B}$, the magnitudes of the AB effect were nearly identical for both digits and symbol targets with letters as distractors. In contrast, when letters were the targets and digits and symbols were the distractors (Experiment 4; see Figure 4A), the $\mathrm{AB}$ effect was much larger for digit distractors than for symbol distractors. The asymmetry is predicted by the dot-level masking account of interference in the $\mathrm{AB}$; symbols are less dense than any of the other three sets and, hence, more dissimilar to letters than are digits or false-font characters (Table 1). This is also true of dissimilarity scores when digits and symbols are treated as targets and letters are treated as distractors. Using data from Experiment 3, we computed the mean dissimilarity scores between each digit and all letters and between each symbol and all letters (the reverse of the computations in Experiment 3 ). The average dissimilarity scores approximated those in Table 1. For visual features, symbol targets were, on average, more dissimilar from letter distractors $(M=11.00)$ than digit targets were dissimilar from letter distractors $(M=$ $10.62)$, but this difference was not significant $[t(14)=$ 0.77]. For pixel densities, symbols were more dissimilar from letters $(M=35.43)$ than were digits $(M=19.25)$, and this difference was significant $[t(14)=4.27]$. Thus, the pattern of results concerning $\mathrm{T}-\mathrm{D}$ similarity effects on the $A B$ appears to be better explained at the level of pixel density than at the level of visual features. 


\section{GENERAL DISCUSSION}

In Experiments 4 and 5, we demonstrated a striking asymmetry. A substantial $A B$ effect was found when mathematical symbols were presented as targets in a stream of letters (Experiment 5), but the AB effect was greatly attenuated when letters were presented as targets in a stream of mathematical symbols (Experiment 4). We also demonstrated symmetry between letters and digits. An $A B$ effect was found both when digits were targets and letters were distractors (Experiment 5) and when letters were targets and digits were distractors (Experiment 4). These effects can be understood as resulting from the properties of our stimulus sets, as was revealed in Experiment 3. Digits and letters both have higher pixel densities than do the mathematical symbols. Thus, digits and letters function as more effective masks than do the symbols. This same analysis may explain the AB effect noted in Experiment 2. In that experiment, we found substantial $\mathrm{AB}$ effects both for word targets presented in a stream of false-font strings and for letter targets presented in a stream of false-font characters. False-font characters appear to function as effective masks because their pixel density is on par with that of letters and digits (Experiment 3). But this line of explanation runs afoul of the results of Experiment 1. If letters and falsefont characters are equally dense and serve as equally effective masks, then why was an $\mathrm{AB}$ effect found for colored word targets appearing in a stream of letter strings, but not for colored word targets appearing in a stream of false-font strings? In the remainder of the discussion, we will address this puzzle by attempting answers to the following three questions. What happens to unattended targets? What is the nature of the processes involved in target identification? And what attracts attention to a potential target?

What is the fate of unattended targets? ${ }^{6}$ Following Chun and Potter (1995), we assume that a target that does not receive attentional (Stage 2) processing is subject to corruption by subsequent distractors. The corrupting effect of post-T2 distractors could occur for multiple reasons, and each finds some empirical support. Distractors could compete with T2 at a conceptual level (see Isaak, Shapiro, \& Martin, 1999) or at a featural level (McAuliffe $\&$ Knowlton, 2000). However, we see little evidence in our results for conceptual interference. The false-font characters were judged to be by far the least familiar and meaningful of our stimuli (Table 1). Consequently, we would expect them to be the least conceptually competitive with letter targets. In contrast, Experiment 2 showed the false-font characters to be quite effective as distractors in producing an $\mathrm{AB}$. Thus, conceptual factors alone cannot explain the pattern of our results. Experiment 3 suggested that lower level factors, at least in the present experimental context, were important. The mathematical symbols, rated nearly as familiar and meaningful as letters, were ineffective as distractors in producing an $\mathrm{AB}$ (Experiment 4). But when the symbols appeared as targets amid letter distractors, a substantial AB effect was observed (Experiment 5). This asymmetry can be explained by differences among our stimuli in low-level properties (pixel density), but not by the differences in higher level properties (familiarity and meaningfulness). However, low-level interference does not explain the differences in $\mathrm{AB}$ magnitude with familiar letters and unfamiliar pattern masks as distractors, reported by other investigators (Isaak et al., 1999). Neither can pixel-level interference alone explain the pattern of results in our Experiment 1. Letters and false-font characters are equally dense (Table 1), yet they had drastically different effects when they appeared as black distractor strings in RSVP streams containing colored words as targets. In order to account for the results of Experiment 1, we have to answer the other two questions.

How is a target processed? When a feature defining a potential target is detected, we assume that an attentional episode is launched in which those objects are processed that appear in a temporal window on the order of $150 \mathrm{msec}$ (see Treisman, 1999, for a similar estimate of attentional dwell time). The hypothesized window is longer than the duration of a single item in these experiments $(100 \mathrm{msec})$, thus accounting for the advantage often shown by the item immediately following T1 (Lag 1 sparing; Visser, Bischof, \& Di Lollo, 1999). But processing of both items within the temporal window implies that some processing must be devoted to discriminating the target from the following item. We follow other investigators of the $\mathrm{AB}$ (Chun \& Potter, 1995; Isaak et al., 1999) in assuming that when the target and the following item share conceptual and/or physical features, discrimination will be more difficult, more attentional resources will be consumed, and more time will be taken by that processing. Thus, for example, discriminating a letter target from a letter distractor will consume more resources and take longer than will discriminating a letter distractor from a false-font character.

What draws attention to a target? In AB experiments, targets are distinguished in some way. Sometimes, the defining feature is categorical, as in searching for letters in digits (Chun \& Potter, 1995, and our Experiment 4). But in other experiments, the defining feature is a change in color and/or luminance. These kinds of changes in elemental stimulus features are responsible, in visual search experiments, for attentional capture (see Yantis, 2000 , for a recent review). Some of the capture may be driven by the properties of the stimulus (such as abrupt onset or changes in color or luminance; e.g., Theeuwes, 1995). Capture also depends on the match between stimulus characteristics and task-dependentattentional control settings (as in the contingent involuntary orienting hypothesis of Folk, Remington, \& Johnston, 1992). Stimulusand goal-driven processes are not mutually exclusive; attention may be captured by a change in a distractor but then quickly redeployed to a possible target (Theeuwes, Atchley, \& Kramer, 2000; Theeuwes \& Godijn, 2001). ${ }^{7}$ We propose, simply, that whatever rules govern attentional capture in experiments on visual search (Theeuwes et al., 2000) and spatial cuing (Folk et al., 1992) also govern 
attentional capture by targets and distractors in RSVP streams. On this view, highlighting a target in an RSVP stream has the effect of directing attention to that target more quickly and, thus, allowing the attentional episode to commence sooner. Note that targets that are not so highlighted by some stimulus change can be discriminated from distractors and identified but that the discrimination is more difficult, takes longer, and becomes efficient only with extended practice (Maki \& Padmanabhan, 1994).

Here, then, is how the concepts of attentional capture and attentional episodes help to account for the pattern of results in Experiment 1. Consider first the case in which distractors are black words (or letters) and targets are colored words (or letters). The instructions emphasize the importance of attending to T1, so we assume the task set contains some rule about priorities that places the color of T1 (red) ahead of the color of T2 (green). Attention captured by $\mathrm{T} 1$ launches an attentional episode. Because the temporal window includes the following distractor, the discrimination demands are high. Because of the high priority of $\mathrm{T} 1$, a delay in responding to the next highlighted word (T2) occurs, and T2 is subjected to masking by subsequent distractors. In the control condition, participants are given a different task set and are instructed to ignore T1. In this case, attention may be initially captured by $\mathrm{T} 1$ but may be quickly redeployed to (or recaptured by) the next highlighted word (see note 7). The same explanation applies to the false-font distractor conditions in Experiment 1, but with the provision that fewer resources are required to discriminate words from false-font strings during the attentional episode, thus allowing spare resources to be applied to identifying T2 when it captures attention. Either T1 is identified extremely quickly or both attentional episodes can run to successful completion even while overlapping each other in time.

Most of our reasoning here is based on the existing literature on attentional variations through time (attentional blink) and space (attentional capture). We have proposed that the concepts associated with attentional capture can help account for the attentional blink. (See Visser et al., 1999, for related ideas about exogenous and endogenous control of the $\mathrm{AB}$.) But to explain the entire pattern of results reported here, we have had to extend Chun and Potter's (1995) model in two ways. First, we have had to assume that attention initially deployed to one potential target can be "recaptured" by a second target appearing closely in time. Thus, whether the processing during an attentional episode runs to completion depends on some top-down, attentional control setting. Second, we have had to assume that two attentional episodes may overlap in time without appreciable interference. Both these assumptions grant considerable flexibility to the processing bottleneck thought to be responsible for the $\mathrm{AB}$ and theoretically localized at Chun and Potter's Stage 2. We follow Jolicœur, Dell'Acqua, and Crebolder (2001) in proposing a functional bottleneck instead of an all-or-none bottleneck. We doubt whether either of these assumptions would be found surprising in the domain of visual search (e.g., the rapid disengagement seen by Theeuwes et al., 2000). But it remains for future research to sort out the implications for our understanding of the $\mathrm{AB}$ and the possibility that it can be triggered by attentional capture.

\section{REFERENCES}

BERINGER,J. (1994). ERTS: A flexible software tool for developing and running psychological reaction time experiments on IBM PCs. Behavior Research Methods, Instruments, \& Computers, 26, 368-372.

Brehaut, J. C., Enns, J. T., \& Di Lollo, V. (1999). Visual masking plays two roles in the attentional blink. Perception \& Psychophysics, 61, 1436-1448.

Broadbent, D. E., \& Broadbent, M. H. P. (1987). From detection to identification: Response to multiple targets in rapid serial visual presentation. Perception \& Psychophysics, 42, 105-113.

Brown, T. L., Roos-Gilbert, L., \& CARr, T. H. (1995). Automaticity and word perception: Evidence from Stroop and Stroop dilution effects. Journal of Experimental Psychology: Learning, Memory, \& Cognition, 21, 1395-1411.

Chun, M. M. (1997). Temporal binding errors are redistributed by the attentional blink. Perception \& Psychophysics, 59, 1191-1199.

Chun, M. M., \& PotTer, M. C. (1995). A two-stage model for multiple target detection in rapid serial visual presentation. Journal of Experimental Psychology: Human Perception \& Performance, 21, 109-127.

Di Lollo, V., Enns, J. T., \& Rensick, R. A. (2000). Competition for consciousness among visual events: The psychophysics of reentrant visual processes. Journal of Experimental Psychology: General, 129, 481-507.

Duncan, J., Ward, R, \& Shapiro, K. (1994). Direct measurement of attentional dwell time in human vision. Nature, 369, 313-315.

Folk, C. L., Remington, R. W., \& Johnston, J. C. (1992). Involuntary covert orienting is contingent on attentional control settings. Journal of Experimental Psychology: Human Perception \& Performance, 18, 1030-1044.

Giesbrecht, B., \& Di Lollo, V. (1998). Beyond the attentional blink: Visual masking by object substitution. Journal of Experimental Psychology: Human Perception \& Performance, 24, 1454-1466.

Grandison, T. D., Ghirardelli, T. G., \& Egeth, H. E. (1997). Beyond similarity: Masking of the target is sufficient to cause the attentional blink. Perception \& Psychophysics, 59, 266-274.

IsAaK, M. I., ShaPIRo, K. L., \& MARTIN, J. (1999). The attentional blink reflects retrieval competition among multiple rapid serial visual presentation items: Tests of an interference model. Journal of Experimental Psychology: Human Perception \& Performance, 25, 1774-1792.

JIANG, Y., \& CHUN, M. M. (2001). The influence of temporal selection on spatial selection and distractor interference: An attentional blink study. Journal of Experimental Psychology: Human Perception \& Performance, 27, 664-679.

JoLICCEUR, P. (1998). Modulation of the attentional blink by on-line response selection: Evidence from speeded and unspeeded Task decisions. Memory \& Cognition, 26, 1014-1032.

Jolicceur, P., Dell'Acqua, R, \& Crebolder, J. M. (2001). The attentional blink bottleneck. In K. Shapiro (Ed.), The limits of attention: Temporal constraints in human information processing (pp. 8299). Oxford: Oxford University Press.

Maki, W. S., Couture, T., Frigen, K., \& Lien, D. (1997). Sources of the attentional blink during rapid serial visual presentation: Perceptual interference and retrieval competition. Journal of Experimental Psychology: Human Perception \& Performance, 23, 1393-1411.

Maki, W. S., Frigen, K., \& PAulson, K. (1997). Associative priming by targets and distractors during rapid serial visual presentation: Does word meaning survive the attentional blink? Journal of Experimental Psychology: Human Perception \& Performance, 23, 1014-1034.

Maki, W. S., \& Padmanabhan, G. (1994). Transient suppression of processing during rapid serial visual presentation: Acquired distinctiveness of probes modulates the attentional blink. Psychonomic Bulletin \& Review, 1, 499-504. 
McAuliffe, S. P., \& Knowlton, B. J. (2000). Dissociating the effects of featural and conceptual interference on multiple target processing in rapid serial visual presentation. Perception \& Psychophysics, 62, 187-195.

McClelland, J. L., \& Rummelhart, D. E. (1981). An interactive activation model of context effects in letter perception: Pt. 1. An account of basic findings. Psychological Review, 88, 375-407.

Nelson, D. L., McEvoy, C. L., \& Schreiber, T. A. (1998). The University of South Florida word association, rhyme, and word fragment norms. Available at http://www.usf.edu/FreeAssociation/.

Petersen, S. E., Fox, P. T., Snyder, A. Z, \& Raichle, M. E. (1990). Activation of extrastriate and frontal cortical areas by visual words and word-like stimuli. Science, 249, 1041-1044.

PotTer, M. C. (1993). Very short-term conceptual memory. Memory \& Cognition, 21, 156-161.

Raymond, J. E., Shapiro, K. L., \& Arnell, K. M. (1992). Temporary suppression of visual processing in an RSVP task: An attentional blink? Journal of Experimental Psychology: Human Perception \& Performance, 18, 849-860.

Seiffert, A. E., \& Di Lollo, V. (1997). Low-level masking in the attentional blink. Journal of Experimental Psychology: Human Perception \& Performance, 23, 1061-1073.

Shapiro, K. L., Caldwell, J., \& Sorensen, R. E. (1997). Personal names and the attentional blink: A visual "cocktail party" effect. Journal of Experimental Psychology: Human Perception \& Performance, 23, 504-514.

Shapiro, K. L., Driver, J., Ward, R., \& Sorensen, R. E. (1997). Priming from the attentional blink: A failure to extract visual tokens but not visual types. Psychological Science, 8, 95-100.

Theeuwes, J. (1995). Abrupt luminance change pops out; abrupt color change does not. Perception \& Psychophysics, 57, 637-644.

Theeuwes, J., Atchley, J., \& Kramer, A. F. (2000). On the timecourse of top-down and bottom-up control of visual attention. In S. Monsell \& J. Driver (Eds.), Control of cognitive processes: Attention and performance XVIII (pp. 105-124). Cambridge, MA: MIT Press.

Theeuwes, J., \& Godisn, R. (2001). Attentional and oculomotor capture. In C. Folk \& B. Gibson (Eds.), Attraction, distraction, and action: Multiple perspectives on attentional capture (pp. 121-149). Amsterdam: Elsevier.

Treisman, A. (1999). Feature binding, attention, and object perception. In G. W. Humphreys, J. Duncan, \& A. Treisman (Eds.), Attention, space, and action: Studies in cognitive neuroscience (pp. 91-111). Oxford: Oxford University Press.

Visser, T. A. W., Bischof, W. F., \& Di Lollo, V. (1999). Attentional switching in spatial and nonspatial domains: Evidence from the attentional blink. Psychological Bulletin, 125, 458-469.

Vogel, E. K., \& LuCK, S. J. (2002). Delayed working memory consolidation during the attentional blink. Psychonomic Bulletin \& Review, 9, 739-743.

Vogel, E. K., Luck, S. J., \& Shapiro, K. L. (1998). Electrophysiological evidence for a postperceptual locus of suppression during the attentional blink. Journal of Experimental Psychology: Human Perception \& Performance, 24, 1656-1674.

Weichselgartner,E., \& Sperling, G. (1987). Dynamics of automatic and controlled visual attention. Science, 238, 778-780.

YANTIS, S. (2000). Goal-directed and stimulus-driven determinants of attentional control. In S. Monsell \& J. Driver (Eds.), Control of cognitive processes: Attention and performance XVIII (pp. 73-103). Cambridge, MA: MIT Press.

\section{NOTES}

1. The U-shaped, or check-shaped, function relating T2 performance to lag is not always found. Performance at Lag 1 appears to be spared when the items in the RSVP stream all occur at the same spatial location and have similar task requirements. When targets appear in different locations, or when targets change with respect to modality or task demands, Lag 1 sparing is often not observed, and the function is monotonic rather than U-shaped (Visser, Bischof, \& Di Lollo, 1999).

2. Isaak, Shapiro, and Martin (1999, Experiment 3) presented pattern masks after some of their targets in five-item RSVP streams. The pattern masks were created by rearranging features of the letters that preceded them $(\mathrm{M}, \mathrm{N}, \mathrm{Z})$. Isaak et al. reported that substituting these masks for letters reduced the magnitude of the $\mathrm{AB}$. This result, which was published shortly after we initiated our Experiments 1 and 2, supports the prediction that our false-font characters should reduce the $\mathrm{AB}$ for letter targets just as they do for word targets.

3. The false-font characters in Figure 1A have a compelling phenomenological appearance of uniqueness, tending toward being very different from the other character sets by virtue of their lack of meaningfulness and lack of familiarity. But conclusions based on our intuitions about these stimuli can be quite misleading. For example, an anonymous reviewer of a previous version of this report claimed that differences among the stimulus categories in Figure 1 "are obvious by inspection." That judgment apparently led the reviewer to the conclusion that "the meaningfulness and familiarity of letters ... [make] letters better targets and more interfering distractors regardless of their visual properties" (personal communication, November 13, 2001). This is a case in which casual inspection leads to an error of introspection. As we shall show (in Experiments 3-5), the similarities of targets and distractors based on their visual properties are better predictors of T-D similarity effects than are meaningfulness and familiarity.

4. After completion of these experiments, we obtained a Minolta LS100 luminance meter and measured the luminance of each character used in these experiments. The meter was mounted on a tripod in the position of a participant's head ( $52 \mathrm{~cm}$ from the display). If the black letters are taken as a background, the average contrast ratios of the red and green target letters $\left(L_{\text {target }} / L_{\text {background }}\right)$ were 1.09 and 1.34 , respectively. Thus, the green targets (T2) were sufficiently luminous to make them "pop out" of visual displays (Theeuwes, 1995).

5. We thank Pierre Jolicœur for suggesting Experiment 5.

6. Our explanations focus on the masking of T2 by $\mathrm{T} 2+1$. However, we admit that our experiments were not designed to separately estimate the effects of the T1 +1 and T $2+1$ distractors (see Chun \& Potter, 1995, and McAuliffe \& Knowlton, 2000) for relevant experiments. Neither were the experiments designed to separate the effects of the distractor immediately following T2 from effects of the overall stream. Sorting out local from global distractor effects is under debate and awaits further research (Isaak et al., 1999).

7. Theeuwes et al. (2000) estimated $150 \mathrm{msec}$ as the time needed for disengagement of attention from a capturing stimulus and redeployment to another spatial location. In the present experiments, in which distractors and targets appeared at only one location, the time for disengagement and redeployment may have been much shorter.

(Manuscript received December 21, 2001; revision accepted for publication June 29, 2002.) 